\title{
Cognitive behavioral training reverses the effect of pain exposure on brain-network activity
}

Article

Accepted Version

Kucyi, A., Salomons, T. V. and Davis, K. D. (2016) Cognitive behavioral training reverses the effect of pain exposure on brain-network activity. Pain, 157 (9). pp. 1895-1904. ISSN 0304-3959 doi:

https://doi.org/10.1097/j.pain.0000000000000592 Available at https://centaur.reading.ac.uk/66024/

It is advisable to refer to the publisher's version if you intend to cite from the work. See Guidance on citing.

To link to this article DOI: http://dx.doi.org/10.1097/j.pain.0000000000000592

Publisher: International Association for the Study of Pain

All outputs in CentAUR are protected by Intellectual Property Rights law, including copyright law. Copyright and IPR is retained by the creators or other copyright holders. Terms and conditions for use of this material are defined in the End User Agreement.

www.reading.ac.uk/centaur 
Central Archive at the University of Reading

Reading's research outputs online 


\title{
Cognitive behavioral training reverses the effect of pain exposure on brain-network activity
}

\author{
Aaron Kucyi* ${ }^{1,3,5,6}$, Tim V. Salomons ${ }^{* 1,7}$, Karen D. Davis ${ }^{1,2,3,4}$
}

${ }^{1}$ Division of Brain, Imaging and Behaviour - Systems Neuroscience, Krembil Research Institute, and ${ }^{2}$ Department of Medical Imaging, University Health Network; ${ }^{3}$ Institute of Medical Science, University of Toronto, ${ }^{4}$ Mount Sinai Hospital Dental Clinic, Toronto, ON, Canada; ${ }^{5}$ Department of Psychiatry, Harvard Medical School, Boston, MA, USA ${ }^{6}$ Department of Psychiatry, Massachusetts General Hospital, Boston, MA, USA ${ }^{7}$ School of Psychology and Clinical Language Sciences, University of Reading, UK

* Authors contributed equally

\section{Number of pages: 35 Number of figures: 7}

Number of tables: 0

\section{Correspondence to:}

Karen D. Davis, Ph.D.

Division of Brain, Imaging and Behaviour - Systems Neuroscience

Toronto Western Research Institute

Toronto Western Hospital, University Health Network 399 Bathurst Street, Room MP14-306

Toronto, Ontario, Canada M5T 2S8

(416) 603-5662 ph; (416) 603-5745 fax

Email: kdavis@uhnres.utoronto.ca

Key Words: pain; cbt; fMRI; default mode network; functional connectivity; cognitive training 


\begin{abstract}
Repeated sensory exposures shape the brain's function and its responses to environmental stimuli. An important clinical and scientific question is how exposure to pain affects brain network activity and whether that activity is modifiable with training. We sought to determine whether repeated pain exposure would impact brain-network activity and whether these effects can be reversed by cognitive behavioral therapy-based training (CBT). Healthy subjects underwent 8 experimental sessions on separate days where they received painful thermal stimuli. They were randomly assigned to groups receiving either CBT-based training (Regulate group, $\mathrm{n}=17$ ) or a non-pain-focused treatment (Control group, $n=13$ ). Before and after these sessions, participants underwent functional MRI (fMRI) during painful stimulation and at rest. The effect of repeated pain over time in the Control group was a decrease in the neurotypical pain-evoked default mode network (DMN) deactivation. The Regulate group did not show these DMN effects but rather had decreased deactivation of the right ventrolateral prefrontal cortex (R vlPFC) of the executive control network. In the Regulate group, reduced pain-evoked DMN deactivation was associated with greater individual reduction in pain intensity and unpleasantness over time. Finally, the Regulate group showed enhanced resting functional connectivity between areas of the DMN and executive control network over time, compared to the Control group. Our study demonstrates that trainable cognitive states can alter the effect of repeated sensory exposure on the brain. The findings point to the potential utility of cognitive training to prevent changes in brain network connectivity that occur with repeated pain experience.
\end{abstract}




\section{Summary}

Eight sessions of cognitive-behavioral training diminishes the effects of repeated noxious stimulation on the brain's default mode network (DMN) response during pain. 


\section{Introduction}

Repeated sensory exposure shapes the brain's function and its responses to exogenous stimuli. For example, repeated exposure to pain can alter the neural response to subsequent pain, potentially contributing to development of chronic pain $[6,19]$. However, pain and its neural representation are strongly influenced by cognitive context $[15,26,53]$, raising the possibility that cognitive training could alter the deleterious effects of repeated pain exposure on the brain. The effects of such training on brain activity however, are poorly understood.

Examination of how pain reshapes brain networks has traditionally focused on sensory and antinociceptive brain systems. However recent neuroimaging evidence suggests a more widespread system at play, referred to as the 'pain connectome,' a spatiotemporal signature comprised of a broader set of interacting networks, including key roles of cognitive networks in association cortices [26]. For example, the default mode (DMN), salience and fronto-parietal/executive control networks underpin dynamic pain-cognition interactions. Functional organization of networks within the pain connectome is reshaped with repeated exposure to acute pain, clinical improvement in chronic pain and the transition from subacute to chronic pain $[6,19,33,37]$. This suggests that a treatment that alters the cognitive context in which pain occurs might alter maladaptive network changes occurring in response to repeated pain. 
Cognitive behavioral therapy (CBT) is a structured psychotherapeutic intervention that targets maladaptive cognitive factors to reduce negative affect [7]. While originally developed for depression, CBT has been successfully adapted to treat pain [47, 48, 54]. Therefore CBT is well suited to investigate experience-dependent dynamics of pain perception and processing in the brain.

Longitudinal neuroimaging studies provide insight into the roles of structural and functional brain regions and networks in CBT for chronic pain [22, 42, 44]. However, a challenge in chronic pain studies is controlling for individual variability in the frequency and intensity of pain over the course of CBT treatment. Furthermore, these studies recruit patients after pain has already become chronic. Studying the effects of repeated pain exposure on brain network activity could provide insight into processes that contribute to chronification and how these changes might be reversed with early intervention.

Here, using a unique, controlled experimental paradigm in healthy subjects, involving repeated exposure to pain over many sessions [39], we assessed the effects of CBT-based training on both pain-evoked brain activity and intrinsic network organization with fMRI. We hypothesized that repeated pain exposure would alter brain-network activity during pain and that those activity alterations could be reversed by CBT-based training. Using an identical level of subjectively reported pain before and after training, we aimed to uncover functional plasticity in the neural processing of pain following pain exposure and cognitive training. Additionally, given that evoked brain-network response patterns show strong similarities with the functional architecture of spontaneous activity [17, 45], we 
also expected that CBT-based training would change intrinsic organization of similar cognitive networks assessed with resting-state fMRI.

\section{Methods}

\section{Subjects}

The experimental manipulation has been described in detail elsewhere [39]. In brief, 34 healthy, pain-free, right-handed individuals (ages 21-38) each randomly assigned to either a CBT-based training or Control group underwent 10 experimental sessions over 21 days that consisted of 8 test sessions and an initial and final session of fMRI during evoked pain and resting-state, and 8 test sessions (see below) (Figure 1). All subjects provided informed consent to procedures approved by the University Health Network research ethics board. Behavioral, but not neuroimaging data, from this dataset were previously reported [39]. At baseline the two groups did not differ in pain-related attitudes, coping strategies, personality factors, or psychological factors such as depression and anxiety (see [39] for details). Four individuals in the Control group (see below) were excluded from the present analyses due to excessive and/or stimuluscorrelated motion in one or more scans (two subjects) or lack of completion of all pain provocation scans at the final session (two subjects). One subject completed the pain provocation but not resting-state scan at the final session. Therefore 13 Control and 17 Regulator subjects were included in pain-evoked fMRI analyses, whereas 12 Control and 17 Regulator subjects were included in resting-state analyses. 


\section{Thermal Detection and Pain Thresholds}

Thermal stimuli were delivered to the left volar forearm with a 30x30mm Peltier thermode (TSA-II,,Medoc Ltd., Israel).

Determination of Intensity of Experimental Stimuli: We used an iterative procedure based on each participant's pain threshold to determine individualized pain levels. Pain thresholds were determined using a ramp procedure (baseline $32^{\circ} \mathrm{C}$, ramp rate $1^{\circ} \mathrm{C} / \mathrm{s}, 30 \mathrm{~s}$ inter-stimulus interval). The threshold was calculated as the average of the final 3 of 4 total trials. Participants were asked to rate 8 s stimuli beginning at pain threshold $+1^{\circ} \mathrm{C}$. If that temperature was rated $\geq 6 / 10$ (numerical rating scale: $0=$ "no pain", $10=$ "worst pain imaginable") on 6 consecutive trials, it was used for further testing. If not, the temperature was raised by $0.5^{\circ} \mathrm{C}$ until that threshold was met (maximum temperature $\leq 50^{\circ} \mathrm{C}$ ). The temperature determined during this calibration phase was then used throughout training. In each of the subsequent $1 \mathrm{hr}$ test sessions, participants received 45 8-s noxious heat stimuli (30s ISI). Participants rated stimulus intensity and unpleasantness every 15 trials $(0-10$ numerical rating scale; $0=$ "no pain", $10=$ "most intense pain imaginable"; $0=$ "not unpleasant", $10=$ "extremely unpleasant"). Between session changes were analyzed by averaging within-session ratings.

The calibration procedure was repeated prior to the final scan session to adjust for habituation and other sources of variation occurring over the course of training, so that the stimulus used in the second scan run was perceptually matched to the one used in the 
first scan. Matching pain intensity across scanning sessions allowed us to investigate how the neural processing of an identical pain percept may change over time, and how CBTbased training may affect the change in neural processing.

\section{Cognitive behavioral training protocol}

Following the first scan but prior to the beginning of training, participants were randomized into one of two conditions. Participants in the Regulate group ( $\mathrm{n}=17,8$ females) were given a brief ( $\sim 5 \mathrm{~min})$ session of pain-focused cognitive behavioural training based on an existing treatment manual (Thorn, 2004) prior to administration of the thermal stimuli. The aim of this training was to reduce their response to the painful stimuli by identifying negative thoughts, reappraising their situation and focus on benefits of the training (e.g. financial compensation, development of pain-coping skills). Participants in this group were educated in the relationship between different aspects of pain (cognitive, emotional and sensory; e.g. "Pain is a major stressor. It can and does produce biological, emotional and cognitive stress responses") and engaged in interactive discussion about their responses to the experimental stimulus and how they might modulate these responses (e.g. "How did your strategy work? Were there any barriers to your being able to successfully suppress the pain?"). Participants in the Control group, ( $\mathrm{n}=13,5$ females) were trained in interpersonal effectiveness (Linehan, 1993). The aim of this training was to improve interpersonal assertiveness and communications with others. Like training in the Regulate group, this training involved both a psychoeducational component ("Just as it is difficult to balance priorities and demands in 
our lives, it can be difficult to balance 'wants' and 'shoulds"'). and interactive discussion of how to implement the material in daily life (e.g. "How effective is the balance between "wants" and "shoulds" in your life? What are some factors that prevent the balance from being more effective?"). Although participants in both groups received equivalent training (both in terms of the amount of time and the format), the Regulate group was encouraged to apply their training during the painful stimuli, but the Control group was not. Text describing these interventions is provided as supplemental material in our previously published study [39].

\section{Neuroimaging}

Neuroimaging data was acquired with a 3-Tesla GE system equipped with an eightchannel phased-array head coil. Pain-evoked and resting-state fMRI scans were acquired using an echo-planar pulse imaging (EPI) sequence (repetition time $=2,000 \mathrm{~ms}$, echo time $=25 \mathrm{~ms}$, axial slice thickness $=4 \mathrm{~mm}$, field of view $=20 \times 20 \mathrm{~cm}, 64 \times 64$ matrix,

voxel size of $3.125 \times 3.125 \times 4 \mathrm{~mm}^{3}$ ). We collected 150 volumes (i.e., 5 mins) in each run. Functional runs were registered to $\mathrm{T} 1$ weighted scans $(\mathrm{TR}=7.8 \mathrm{~ms}, \mathrm{TI}=300$, flip angle $=20^{\circ}$, matrix $=134$, FOV $=20$, bandwidth $=15.6$, slice thickness $=1.5 \mathrm{~mm}$, slices $=108$ )

At both sessions, subjects were given 6 painful 8s thermal stimuli at the pre-determined level (see above) delivered to their left volar forearm. There was a 30s ISI, and subjects rated pain intensity and unpleasantness after each stimulus (VAS, same anchors as above). The break between pain offset and rating scale onset was jittered between 8-14 s $($ mean $=11 \mathrm{~s})$. Previous studies indicate that inter-session test-retest reliability in BOLD fMRI activation can be high in several cortical regions for as few as five 15 -sec evoked 
heat painful stimuli (e.g. intra-class correlations ranging from 0.5 to 0.859 across two sessions) [49], and that similarly few trials are needed to detect pain-evoked deactivations [24]. Therefore we believe we likely had sufficient trials to detect reliable effects (see Discussion however).

\section{Pain-evoked brain activity: fMRI preprocessing and analysis}

Using FEAT in FSL v5.0 [21], whole-brain voxel-wise activation/deactivation analyses were conducted on the fMRI scans of painful stimulation that were percept-matched between pre- and post-training scans. Individual runs were preprocessed with the following procedures: first 5 volumes deleted, motion correction (MCFLIRT), brain extraction (BET), spatial smoothing (6mm full-width at half maximum kernel), and highpass temporal filtering $(0.01 \mathrm{~Hz}$ cut-off). Linear registration (FLIRT) was performed among T1, fMRI and MNI152 2x2x2 $\mathrm{mm}$ standard space.

First-level (within-run) general linear model (GLM) analyses in native fMRI space were conducted with FILM prewhitening and with two separate regressors (pain stimulation period and pain rating period), each convolved with a gamma hemodynamic response function and with temporal filtering applied. A first-level contrast was set up to create voxel-wise contrast of parameter estimate (COPE) maps of pain-evoked activation. These maps were passed on as inputs to a second-level (within-subject) analysis of the two time points. The maps were converted to MNI152 space, and fixed effects analyses were performed with 4 contrasts to identify pain-evoked activation: time 1 alone, time 2 alone, time $1>$ time 2 and time $2>$ time 1 . The resulting maps for each contrast were then 
entered into a third-level (group-level) FLAME2 mixed effects analysis (threshold: cluster based $\mathrm{p}<0.05$; FWE-corrected $\mathrm{Z}>2.3$ ), with 8 contrasts: time 1 activation, time 1 deactivation, time 2 activation, time 2 deactivation, time $2>$ time 1 (each group separately), time $1>$ time 2 (each group separately), time $2>$ time 1 (Control $>$ Regulate group), and time $1>$ time 2 (Regulate $>$ Control group). Thus the main analyses assessing the effect of group on brain activation changes over time were repeated measures (time 1, time 2) ANOVAs with groups (Control, Regulate) as factors.

We next sought to determine whether significant group differences in pain-evoked activation over time were behaviorally relevant at level of individual differences in changes in pain. We thus tested relationships between neural activation and behavior by extracting each individual's mean Z-score values for the appropriate contrast from core regions DMN regions that showed significant group differences in pain-evoked activation from time 1 to time 2 [6mm-diameter spheres drawn around peak coordinates in mPFC $(\mathrm{MNI} x y z=-2,52,14)$ and $\mathrm{PCC} / \mathrm{PCu}(\mathrm{MNI} x y z=4,-54,18)]$. We calculated Pearson correlations between changes in activation in these regions and changes in secondary hyperalgesia (which demonstrated a significant group $\mathrm{x}$ time interaction in our previous analysis [39]), pain intensity and pain unpleasantness over the course of training. We also performed these analyses for a right ventrolateral prefrontal cortex (R vlPFC) region that showed significant group differences in pain-evoked activation from time 1 to 2 [6mm-diameter sphere drawn around peak coordinates $(\mathrm{MNI} x y z=50,42,6)]$. The correlation analyses were thresholded at $\mathrm{p}<0.05$. Given previously identified associations between the DMN and pain-related cognition assessed with the pain catastrophizing scale 
(PCS) $[20,28,46]$, we also correlated DMN activity changes with in PCS at time 2 compared to time 1 in both groups (although one Control subject had missing PCS scores and was not included).

Resting-state fMRI: preprocessing and analysis

Longitudinal analyses of resting-state fMRI data were performed to test hypotheses that were based on between-group differences that were identified from the pain-evoked activation analyses. In line with the notion that stimulus-/task-evoked activity patterns show strong similarities with the functional architecture of spontaneous activity [17], we expected that key regions showing group differences in pain-evoked activation due to CBT-based training would also show related effects on resting state connectivity. Thus, seed regions for resting-state fMRI analysis (described below) were defined based on pain-activation differences between groups.

Resting-state data were preprocessed as done previously [25, 28, 29], using a combination of FSL, MATLAB v.7.12.0 (Mathworks), and fMRISTAT [56]. Firstly, using FSL's FEAT, the following was done: deletion of the first 4 volumes, motion correction (MCFLIRT), brain extraction (BET), and linear registration among fMRI, T1 and standard MNI152 2x2x2 mm space. The T1 scans were segmented into gray matter, white matter (WM), and cerebrospinal fluid (CSF) using FSL's FAST. The WM and CSF partial volume maps were then registered to fMRI space and were thresholded to retain the peak voxels constituting volumes of $198 \mathrm{~cm}^{3}$ for WM and $20 \mathrm{~cm}^{3}$ for CSF [13]. Following aCompCor procedures for removal of physiological and scanner-related noise 
[8], principal components analysis was performed on the fMRI data within the WM and CSF masks separately. The top five WM components, top five CSF components, and six motion parameters that were obtained with MCFLIRT were then regressed from the fMRI data. Finally, spatial smoothing (6mm full-width at half-maximum kernel) and bandpass temporal filtering $(0.005-0.05 \mathrm{~Hz})$ were performed.

A first-level seed-based functional connectivity analysis was conducted with each run, using FEAT. Seed regions were chosen based on the significant group difference map showing effects of CBT-related training on pain-evoked activation. This map contained regions mainly within the DMN but also a lateral prefrontal region (R vlPFC) that had different directionality in group-related activation effects than the DMN regions.

Thus one seed was defined as a $6 \mathrm{~mm}$-diameter sphere surrounding peak coordinates in a location within R vlPFC (MNI xyz $=50,42,6)$ where the Regulate group showed a greater decrease in pain-evoked activation from time 1 to time 2 than the Control group. The seed was registered from MNI to fMRI space, and the mean time course within seed voxels was extracted. This time course was entered as a regressor in a first-level GLM for each run to create voxel-wise COPE maps of functional connectivity. Second-level (within-subject) and third-level (group-level) analyses were conducted following the same procedures and statistical thresholds as in the pain-evoked activation analyses. An identical analysis was repeated for a seed in the $\mathrm{PCC} / \mathrm{PCu}$ region $(6 \mathrm{~mm}$ diameter around peak coordinates: MNI xyz $==4,-54,18)$ that showed significant group differences in pain-evoked activation from time 1 to time 2 (greater decrease in deactivation over time 
in the Control compared to Regulate group). We chose the $\mathrm{PCC} / \mathrm{PCu}$ as the seed, as opposed to other regions within the DMN that showed similar pain activation effects, because the PCC/PCu is considered a hub within the DMN [1].

\section{Results}

\section{Stimulus intensity and pain ratings}

The temperatures [mean (SD)] used to evoke the "perceptually matched ratings" of pain within groups at both time points are listed in Table 1 . There was no Time by group interaction $(\mathrm{F}=0.26, \mathrm{p}=0.61)$ and the groups did not differ significantly at either time point (time $1 \mathrm{~F}=1.25, \mathrm{p}=0.27$; time $2 \mathrm{~F}=2.68, \mathrm{p}=0.11$ ).

As previously reported [39], during training sessions, there was a significant group by time interaction for pain unpleasantness, but not intensity, such that there was a significantly greater reduction in pain unpleasantness in the Regulate group than in the Control group. No such interaction was observed for pain intensity. It should be noted that unlike the stimuli used during the scan session, these stimuli were not delivered at a set perceptual level but, rather, at the level of stimulation used at the initial session.

For pain ratings collected during the scan sessions, we intended to match the groups on pain ratings (see Introduction/Methods) and so we statistically tested whether this was successfully achieved. Mean (SD) pain intensity and unpleasantness ratings at both time 
points within each group are listed in Table 1. Pain intensity ratings at time 2 were not significantly different from those at time 1 for either group (repeated measures ANOVA $\mathrm{F} 1,12=3.37, \mathrm{p}=0.09$ Control, F1,15=0.03, $\mathrm{p}=0.86$ Regulate), and the Time by Group interaction was not significant $(\mathrm{F}=1.4, \mathrm{p}=0.25)$, confirming that stimuli were perceptmatched between sessions. There were no significant group differences in pain intensity at time $1(\mathrm{~F}=2.12, \mathrm{p}=0.16)$ or at time $2(\mathrm{~F}=0.11, \mathrm{p}=0.74)$.

Pain unpleasantness ratings at time 2 were not significantly different from those at time 1 for either group (repeated measures ANOVA F1,12=4.39, $\mathrm{p}=0.06$ Control, F1,15=0.06, $\mathrm{p}=0.81$ Regulate), and the Time by Group interaction was not significant $(\mathrm{F}=1.4, \mathrm{p}=0.24)$. There were no significant group differences in pain unpleasantness at time $1(\mathrm{~F}=1.94$ $\mathrm{p}=0.18)$, or at time $2(\mathrm{~F}=0.18, \mathrm{p}=0.68)$.

\section{Effect of CBT-based training on pain-evoked brain activation}

At time 1 (pre-intervention), both the Regulate and Control groups had pain-evoked activations located within regions of the somatosensory and salience networks including bilateral insula, mid-cingulate cortex (MCC), dorsolateral prefrontal cortex (dlPFC), secondary somatosensory cortex (S2), and caudate nucleus. There were also pain-evoked deactivations in regions of the default mode network (DMN) [bilateral posterior cingulate cortex $(\mathrm{PCC}) /$ precuneus $(\mathrm{PCu})$, medial prefrontal cortex $(\mathrm{mPFC})$, lateral parietal cortex (LPC)] (Figure 2). There were no significant group differences in pain-evoked activity at time 1 (FWE-corrected $\mathrm{Z}>2.3$, cluster-based $\mathrm{p}<0.05$ ). This demonstration, in each group, 
of pre-training whole-brain pain-related activation and deactivation patterns consistent with previous literature $[2,26,32]$, combined with the lack of significant group differences, served to validate our approach and suitability of sample size (i.e., we were powered enough to detect canonical pain-related neural activity in two independent groups with no pre-existing group differences).

Crucially, there were significant group differences in the changes of pain-evoked brain activations from time 1 to time 2 (i.e., the Time by Group interaction was significant) in several regions (see Figure 3A-C). Follow-up within group analyses indicated that the significant Time by Group interaction observed in $\mathrm{DMN}$ regions $(\mathrm{PCC} / \mathrm{PCu}, \mathrm{mPFC}, \mathrm{LPC}$ - Figure 3C) was driven by changes in deactivation in the Control subjects that was not observed in the Regulate group. Specifically, there was deactivation within these DMN regions in both groups at time 1 , but a significant decrease in deactivation at time 2 relative to time 1 (FWE-corrected $\mathrm{Z}>2.3$, cluster-based $\mathrm{p}<0.05)$ was observed in the Control group (see Figure 3A and example plot for $\mathrm{PCC} / \mathrm{PCu}$ in Figure 3C).

A significant Time by Group interaction was also observed in a dorsolateral prefrontal region with peak coordinates in the right ventrolateral prefrontal cortex (R vlPFC) but follow up analyses revealed that the pattern of change for this region differed from the DMN. There was no activation in either group at time 1 but significant increase in deactivation in the Regulate (FWE-corrected $\mathrm{Z}>2.3$, cluster-based $\mathrm{p}<0.05$ ) but not Control group at time 2 compared to time 1 (see Figure 3B and plot for vlPFC in Figure 3C). 
Brain activation changes over time correlate with individual pain ratings Within the Control group, changes in pain-evoked DMN core (PCC/PCu and mPFC) activity from the time 1 scan (pre-training) to the time 2 scan (post-training) were not correlated with the pain intensity $(\mathrm{r}=-0.09, \mathrm{p}=0.78)$ or pain unpleasantness $(\mathrm{r}=-0.12$, $\mathrm{p}=0.7$ ) changes from the first training session (behavioral session 1 ) to the last (behavioral session 8). However, in the Regulate group, reduced pain-evoked DMN core deactivation from the time 1 scan (pre-training) to the time 2 scan (post-training) was significantly correlated with the reduction in pain intensity $(r=0.54, p=0.025)$ and pain unpleasantness ( $\mathrm{r}=0.52, \mathrm{p}=0.033$ ) from the first training session (behavioral session 1$)$ to the last (behavioral session 8) (Figure 4). Correlations between the groups were compared using a Fisher $\mathrm{r}$ to $\mathrm{z}$ transform. For both intensity and unpleasantness, the correlations were not significantly different between groups at the two-tailed level $(\mathrm{z}=1.68, \mathrm{p}=0.09)$.

In the Regulate group the change in pain-evoked activation in the R vlPFC from scan time 1 to 2 correlated with pain intensity $(r=0.48, p=0.051)$ and pain unpleasantness $(\mathrm{r}=0.5, \mathrm{p}=0.04)$ changes from the first to last training session. There were no similar correlations in the Control group $(\mathrm{r}=0.12, \mathrm{p}=0.71$ for intensity; $\mathrm{r}=0.02, \mathrm{p}=0.96$ for unpleasantness). However, for both intensity and unpleasantness, these R vlPFC activation correlations were not significantly different between groups $(\mathrm{z}=0.98, \mathrm{p}=0.16$ for intensity; $\mathrm{z}=1.29, \mathrm{p}=0.1$ for unpleasantness). There were no significant correlations 
between DMN activity changes and changes in PCS over time in the Regulate group ( $\mathrm{r}=-$ $0.02, \mathrm{p}=0.94)$ or in the Control group $(\mathrm{r}=0.47, \mathrm{p}=0.12)$.

\section{Effect of CBT-based training on resting-state functional connectivity}

The R vlPFC region that demonstrated a significant group differences in response to CBT-based training in the pain-evoked analysis was used as a seed region to assess resting-state functional connectivity changes. This region showed increased pain-evoked deactivation over time in the Regulate but not Control group, so we sought to investigate how its intrinsic network connectivity may have changed differentially between groups. At time 1 (pre training), in both Regulate and Control groups, the R vlPFC exhibited resting-state functional connectivity with a fronto-temporal-parietal network including $\mathrm{R}$ TPJ and L dlPFC (Figure 5), resembling the executive control network [27, 57]. There were no significant group differences in R vlPFC functional connectivity at time 1 (FWEcorrected $\mathrm{Z}>2.3$, cluster-based $\mathrm{p}<0.05$ ).

As in the pain-activation analysis, there were significant group interactions with time for R vlPFC functional connectivity (see Figure 6A-C). The analysis of changes from time 1 to time 2 revealed that a region within $\mathrm{mPFC}$ exhibited a greater increase in functional connectivity with the R vlPFC in the Regulate compared to Control group (Figure 6C) (FWE-corrected $\mathrm{Z}>2.3$, cluster-based $\mathrm{p}<0.05$ ). Within-group functional connectivity results reveal the changes over time that drove the group interaction with time (Figure 6A, 6B). Within the Control group, there was increased $\mathrm{R}$ vlPFC functional connectivity 
at time 2 compared to time 1 with the mid-cingulate cortex (MCC) and an anterior portion of the $\mathrm{PCu}$ (Figure 6A), both of which are regions typically associated with the salience network [57]. In contrast, within the Regulate group, there was increased R vlPFC functional connectivity at time 2 compared to time 1 with the $\mathrm{mPFC}$ and $\mathrm{PCC} / \mathrm{PCu}$, which are regions of the DMN (Figure 6B) (FWE-corrected $\mathrm{Z}>2.3$, clusterbased $\mathrm{p}<0.05)$.

The mPFC cluster that exhibited a group by time interaction effect in the resting state analysis overlapped with $\mathrm{mPFC}$ regions that showed a greater increase in pain-evoked activation in the Control compared to Regulate group at time 2 compared to time 1 (Figure 7). No significant group differences in the change of resting-state functional connectivity over time were found when using the $\mathrm{PCC} / \mathrm{PCu}$ as a seed region.

\section{DISCUSSION}

Here we showed that repeated exposure to pain alters pain-evoked brain activity, specifically by diminishing the normal pain-evoked deactivation of DMN. Importantly, this altered response was reversed by cognitive training. We show that 8 sessions of CBT-based training maintains normal patterns of pain-evoked DMN deactivation, and that the degree to which this DMN deactivation is reduced is associated with decreases in 
pain intensity and unpleasantness over time due to cognitive training. Additionally, cognitive training resulted in decreased right vlPFC activation during painful stimulation and increased spontaneous functional connectivity between the DMN and right vlPFC. This work highlights the dynamic nature of pain and its neural representation, emphasizing a role of cognitive brain networks in training effects on pain perception.

\section{Default mode network (de)activation and pain}

In healthy individuals, the DMN is typically deactivated during acute pain $[14,35,36$, 41], a result that was replicated in both groups in our pre-intervention findings. In our study, repeated pain exposure (in the Control group) eradicated this DMN deactivation but cognitive training (in the Regulate group) maintained it.

One interpretation is that Control subjects, in absence of cognitive training to cope with pain, gradually became 'chronic pain-like' in their DMN responses. Previous studies showed changes in mPFC (of the DMN) activity and connectivity associated with chronic pain development $[6,19]$. Chronic pain patients showed attenuated DMN deactivation during cognitive task performance $[4,11,43,51]$. Furthermore, chronic pain patients showed a lack of DMN deactivation during painful stimulation, a response that was restored with successful analgesic treatment [18]. Also of relevance to our findings, prolonged exposure to uncontrollable (compared to controllable) pain, was shown to elicit greater pain-evoked DMN activation [40]. It is thus possible that pain exposure (chronic or repeated instances of acute pain) results in attenuated pain-evoked DMN 
deactivation, but this attenuation can be prevented/reversed with $\mathrm{CBT}$ or analgesic treatment.

Another possible interpretation is that the observed group differences in brain activation are due to different pain coping strategies. Attenuated DMN deactivation during painful stimulation has been linked to fluctuating attention to pain [29]. Together with our observation of an association between reduced pain-evoked DMN deactivation and reduced pain intensity and unpleasantness in the Regulate group, this suggests that cognitive training might alter attentional focus on pain. Given the previously demonstrated link between vlPFC activation and reappraisal of pain [38, 40,52], it is possible that changes in pain-evoked activation of this region and resting state connectivity between vlPFC and DMN are associated with a process whereby volitional appraisal alters subsequent attention to pain. Further studies with specific measures of reappraisal and attention to pain are needed to confirm these interpretations.

\section{Cognitive-behavioral therapy for pain and the brain}

Longitudinal studies have begun to uncover CBT effects on the brain in chronic pain. Jensen et al. [22] found enhanced pain-evoked vlPFC activation and functional connectivity with the thalamus due to CBT. Seminowicz et al. [42] showed increased gray matter volume following CBT in several regions spanning association (e.g. prefrontal) and sensory (S1/S2) cortices. Shpaner et al. [44] revealed decreased spontaneous interactions between the DMN and antinociceptive regions, and increased connectivity between S2 and basal ganglia due to CBT. 
This study is unique from previous studies in that we studied healthy subjects, exposed all subjects to equal amounts of pain, matched stimuli pre- and post-treatment for pain intensity, and studied both pain-evoked and intrinsic brain activity in Regulate and Control groups. Our paradigm enabled us to specifically attribute effects to CBT-based training, with minimal impact of confounding factors.

We recently showed that this CBT training paradigm reduces secondary hyperalgesia (i.e., a lack of enhanced pain sensitivity beyond the site of stimulus-induced injury) in healthy individuals [39]. Our neuroimaging results here further delineate the biological mechanisms of CBT-based training for pain. Partly consistent with CBT studies of chronic pain [44], our results emphasize a role of the DMN. Further studies with multimodal imaging approaches (such as those applied here) are needed to characterize pain-related and intrinsic activity in chronic pain. Interestingly, neuroimaging studies of CBT for depression have demonstrated effects on the DMN (particularly mPFC) as well as lateral prefrontal areas including vlPFC $[16,23,58]$, possibly indicating common mechanisms between CBT for mood and pain control.

Beyond CBT, neuroimaging studies of multiple, distinct cognitive regulation and reappraisal strategies for pain are revealing potentially specific brain mechanisms for different pain coping strategies [9]. For example, recent studies of acute pain in healthy individuals point toward potentially unique brain mechanisms underlying self-regulation of pain [55] and mindfulness meditation [59]. Thus, an additional area requiring further 
inquiry pertains to how $\mathrm{CBT}$ effects on the brain compare to effects of other cognitive strategies for pain coping.

\section{Clinical implications}

Accumulating evidence implicates abnormal DMN activation and connectivity in the pathophysiology of chronic pain. Chronic pain patients show attenuated DMN deactivation during cognitive performance $[4,43,51]$. Resting-state fMRI studies reveal abnormal functional connectivity within the DMN and between the DMN and other networks in multiple chronic pain disorders [3, 5, 12, 28, 30, 31, 34]. Furthermore, DMN regions show altered interactions with cognitive-, pain- and antinociception-related networks as chronic pain improves over time with diverse treatments $[6,12,33]$. Therefore the DMN may be an important target whose aberrant activity and connectivity may be alleviated with CBT for chronic pain.

CBT (and similar treatments) affect the pain experience by changing beliefs and expectations about pain. As such, we expect some mechanistic overlap with effects like placebo analgesia that work by manipulating pain-related beliefs [50]. A critical difference, from both a scientific and clinical standpoint, however, is that CBT brings these effects under an individual's volitional control. Further longitudinal study of the neural mechanisms through which CBT and other psychotherapeutic interventions work will facilitate enhancement of these effects through training, leading to more efficient and effective treatment for pain. Our paradigm detected changes in pain-evoked neural activation and reversal of these changes by CBT-based training. Although further study 
will be needed to test the applicability of the paradigm to chronic pain populations, it appears promising in terms of better understanding the transition from acute to chronic pain states and how cognitive training can alter neural processes that give rise to this transition.

\section{Limitations}

Our study should be considered preliminary and should ideally be reproduced and built upon in future work with higher sample sizes and greater numbers of trials for measuring pain-evoked brain activity [10]. There was a slight imbalance between the number of Regulator $(\mathrm{n}=17)$ and Control $(\mathrm{n}=13$ for activation analyses, $\mathrm{n}=12$ for resting-state analyses) subjects, which could have introduced statistical power issues that could have partially undermined some of our findings. Our demonstration that the two groups showed canonical, and similar, pain-evoked brain activity as well as resting FC at the whole-brain level at baseline improves our confidence in our study as being sufficiently powerful, but we cannot be certain that longitudinal analyses were unaffected by issues with sample size and/or number of trials.

While we speculate that subjects in the Regulate group each adopted unique strategies based on their cognitive training to cope with pain, we did not include a measure to assess individual differences in strategies. Thus future work is needed to link brain activity changes with specific pain-coping strategies. Furthermore, while our study design with healthy individuals was chosen to limit confounding factors that may be present with a clinical sample, understanding the relevance of our results to chronic pain states 
remains a challenge. It is possible that chronic pain patients would develop different coping strategies in response to cognitive training than would healthy individuals coping with acute pain, and such strategies could be linked with different brain mechanisms than those investigated here.

\section{Conclusion}

Cognitive-behavioral training for pain regulation in healthy individuals changes the brain's typical response to repeated pain exposure. Consistent with findings in the chronic pain literature, we observed alterations in DMN responses with repeated exposure to pain. With CBT-based training, these alterations were ablated, in conjunction with increases in vlPFC responses to pain and increased intrinsic connectivity between vlPFC and DMN. Monitoring brain activity and connectivity before, during and after CBT in clinical settings may facilitate development of effective, personalized pain therapies.

\section{Acknowledgements}

The authors have no conflicts of interest to declare. This work was supported by the Canadian Institutes of Health Research and the Canada Research Chair program. TVS was funded by a Clinician-Scientist Award from the University of Toronto Centre 
for the Study of Pain and a Marie Curie Fellowship from the European Commission. AK was supported by a Canadian Institutes of Health doctoral scholarship. 


\section{References}

1. Andrews-Hanna JR, Reidler JS, Sepulcre J, Poulin R, and Buckner RL. Functional-anatomic fractionation of the brain's default network. Neuron 2010;65(4):550-62.

2. Apkarian AV, Bushnell MC, Treede RD, and Zubieta JK. Human brain mechanisms of pain perception and regulation in health and disease. Eur.J.Pain 2005;9(4):463-484.

3. Baliki MN, Baria AT, and Apkarian AV. The cortical rhythms of chronic back pain. J Neurosci 2011;31(39):13981-90.

4. Baliki MN, Geha PY, Apkarian AV, and Chialvo DR. Beyond feeling: chronic pain hurts the brain, disrupting the default-mode network dynamics. J Neurosci 2008;28(6):1398-403.

5. Baliki MN, Mansour AR, Baria AT, and Apkarian AV. Functional Reorganization of the Default Mode Network across Chronic Pain Conditions. PLoS One 2014;9(9):e106133.

6. Baliki MN, Petre B, Torbey S, Herrmann KM, Huang L, Schnitzer TJ, Fields HL, and Apkarian AV. Corticostriatal functional connectivity predicts transition to chronic back pain. Nat Neurosci 2012;15(8):1117-9.

7. Beck A, Cognitive therapy of depression. 1979, New York: Guilford Press.

8. Behzadi Y, Restom K, Liau J, and Liu TT. A component based noise correction method (CompCor) for BOLD and perfusion based fMRI. Neuroimage 2007;37(1):90-101.

9. Bushnell MC, Ceko M, and Low LA. Cognitive and emotional control of pain and its disruption in chronic pain. Nat Rev Neurosci 2013;14(7):502-11.

10. Button KS, Ioannidis JP, Mokrysz C, Nosek BA, Flint J, Robinson ES, and Munafo MR. Power failure: why small sample size undermines the reliability of neuroscience. Nat Rev Neurosci 2013;14(5):365-76.

11. Ceko M, Gracely JL, Fitzcharles MA, Seminowicz DA, Schweinhardt P, and Bushnell MC. Is a Responsive Default Mode Network Required for Successful Working Memory Task Performance? J Neurosci 2015;35(33):11595-605.

12. Ceko M, Shir Y, Ouellet JA, Ware MA, Stone LS, and Seminowicz DA. Partial recovery of abnormal insula and dorsolateral prefrontal connectivity to cognitive networks in chronic low back pain after treatment. Hum Brain Mapp 2015.

13. Chai XJ, Castanon AN, Ongur D, and Whitfield-Gabrieli S. Anticorrelations in resting state networks without global signal regression. Neuroimage 2012;59(2):1420-8.

14. Coghill RC, Talbot JD, Evans AC, Meyer E, Gjedde A, and Bushnell MC. Distributed processing of pain and vibration by the human brain. Journal of Neuroscience 1994;14:4095-4108.

15. Eccleston $\mathrm{C}$ and Crombez G. Pain demands attention: a cognitive-affective model of the interruptive function of pain. Psychol Bull 1999;125(3):356-66.

16. Goldapple K, Segal Z, Garson C, Lau M, Bieling P, Kennedy S, and Mayberg H. Modulation of cortical-limbic pathways in major depression: treatment-specific effects of cognitive behavior therapy. Arch Gen Psychiatry 2004;61(1):34-41. 
17. Harmelech $\mathrm{T}$ and Malach R. Neurocognitive biases and the patterns of spontaneous correlations in the human cortex. Trends Cogn Sci 2013;17(12):60615.

18. Harris RE, Napadow V, Huggins JP, Pauer L, Kim J, Hampson J, Sundgren PC, Foerster B, Petrou M, Schmidt-Wilcke T, and Clauw DJ. Pregabalin rectifies aberrant brain chemistry, connectivity, and functional response in chronic pain patients. Anesthesiology 2013;119(6):1453-64.

19. Hashmi JA, Baliki MN, Huang L, Baria AT, Torbey S, Hermann KM, Schnitzer TJ, and Apkarian AV. Shape shifting pain: chronification of back pain shifts brain representation from nociceptive to emotional circuits. Brain 2013;136(Pt 9):275168.

20. Hubbard CS, Khan SA, Keaser ML, Mathur VA, Goyal M, and Seminowicz DA. Altered Brain Structure and Function Correlate with Disease Severity and Pain Catastrophizing in Migraine Patients. eNeuro 2014;1(1):e20 14.

21. Jenkinson M, Beckmann CF, Behrens TE, Woolrich MW, and Smith SM. Fsl. NeuroImage 2012;62(2):782-90.

22. Jensen KB, Kosek E, Wicksell R, Kemani M, Olsson G, Merle JV, Kadetoff D, and Ingvar M. Cognitive Behavioral Therapy increases pain-evoked activation of the prefrontal cortex in patients with fibromyalgia. Pain 2012;153(7):1495-503.

23. Kennedy SH, Konarski JZ, Segal ZV, Lau MA, Bieling PJ, McIntyre RS, and Mayberg HS. Differences in brain glucose metabolism between responders to CBT and venlafaxine in a 16-week randomized controlled trial. Am J Psychiatry 2007;164(5):778-88.

24. Koyama T, McHaffie JG, Laurienti PJ, and Coghill RC. The single-epoch fMRI design: validation of a simplified paradigm for the collection of subjective ratings. Neuroimage 2003;19(3):976-987.

25. Kucyi A and Davis KD. Dynamic functional connectivity of the default mode network tracks daydreaming. NeuroImage 2014;100C:471-480.

26. Kucyi A and Davis KD. The dynamic pain connectome. Trends Neurosci 2015;38(2):86-95.

27. Kucyi A, Hodaie M, and Davis KD. Lateralization in intrinsic functional connectivity of the temporoparietal junction with salience- and attention-related brain networks. J Neurophysiol 2012;108(12):3382-92.

28. Kucyi A, Moayedi M, Weissman-Fogel I, Goldberg MB, Freeman BV, Tenenbaum HC, and Davis KD. Enhanced medial prefrontal-default mode network functional connectivity in chronic pain and its association with pain rumination. J Neurosci 2014;34(11):3969-75.

29. Kucyi A, Salomons TV, and Davis KD. Mind wandering away from pain dynamically engages antinociceptive and default mode brain networks. Proc Natl Acad Sci U S A 2013;110(46):18692-7.

30. Loggia ML, Kim J, Gollub RL, Vangel MG, Kirsch I, Kong J, Wasan AD, and Napadow V. Default mode network connectivity encodes clinical pain: an arterial spin labeling study. Pain 2013;154(1):24-33.

31. Martucci KT, Shirer WR, Bagarinao E, Johnson KA, Farmer MA, Labus JS, Apkarian AV, Deutsch G, Harris RE, Mayer EA, Clauw DJ, Greicius MD, and Mackey SC. The Posterior Medial Cortex in Urologic Chronic Pelvic Pain 
Syndrome: Detachment from Default Mode Network. A Resting-State Study from the MAPP Research Network. Pain 2015.

32. Mouraux A, Diukova A, Lee MC, Wise RG, and Iannetti GD. A multisensory investigation of the functional significance of the "pain matrix". Neuroimage. 2011;54(3):2237-2249.

33. Napadow V, Kim J, Clauw DJ, and Harris RE. Decreased intrinsic brain connectivity is associated with reduced clinical pain in fibromyalgia. Arthritis Rheum 2012;64(7):2398-403.

34. Napadow V, LaCount L, Park K, As-Sanie S, Clauw DJ, and Harris RE. Intrinsic brain connectivity in fibromyalgia is associated with chronic pain intensity. Arthritis Rheum. 2010;62(8):2545-2555.

35. Owen DG, Clarke CF, Ganapathy S, Prato FS, and St Lawrence KS. Using perfusion MRI to measure the dynamic changes in neural activation associated with tonic muscular pain. Pain 2010;148(3):375-86.

36. Peyron R, Garcia-Larrea L, Gregoire MC, Costes N, Convers P, Lavenne F, Mauguiere F, Michel D, and Laurent B. Haemodynamic brain responses to acute pain in humans: sensory and attentional networks. Brain 1999;122 (Pt 9):17651780.

37. Riedl V, Valet M, Woller A, Sorg C, Vogel D, Sprenger T, Boecker H, Wohlschlager AM, and Tolle TR. Repeated pain induces adaptations of intrinsic brain activity to reflect past and predict future pain. NeuroImage 2011;57(1):20613.

38. Salomons TV, Johnstone T, Backonja MM, Shackman AJ, and Davidson RJ. Individual differences in the effects of perceived controllability on pain perception: critical role of the prefrontal cortex. J.Cogn Neurosci. 2007;19(6):993-1003.

39. Salomons TV, Moayedi M, Erpelding N, and Davis KD. A brief cognitivebehavioural intervention for pain reduces secondary hyperalgesia. Pain 2014;155(8):1446-52.

40. Salomons TV, Nusslock R, Detloff A, Johnstone T, and Davidson RJ. Neural emotion regulation circuitry underlying anxiolytic effects of perceived control over pain. J Cogn Neurosci 2015;27(2):222-33.

41. Seminowicz DA and Davis KD. Pain enhances functional connectivity of a brain network evoked by performance of a cognitive task. J Neurophysiol 2007;97(5):3651-9.

42. Seminowicz DA, Shpaner M, Keaser ML, Krauthamer GM, Mantegna J, Dumas JA, Newhouse PA, Filippi CG, Keefe FJ, and Naylor MR. Cognitive-behavioral therapy increases prefrontal cortex gray matter in patients with chronic pain. $\mathrm{J}$ Pain 2013;14(12):1573-84.

43. Seminowicz DA, Wideman TH, Naso L, Hatami-Khoroushahi Z, Fallatah S, Ware MA, Jarzem P, Bushnell MC, Shir Y, Ouellet JA, and Stone LS. Effective treatment of chronic low back pain in humans reverses abnormal brain anatomy and function. J Neurosci 2011;31(20):7540-50.

44. Shpaner M, Kelly C, Lieberman G, Perelman H, Davis M, Keefe FJ, and Naylor MR. Unlearning chronic pain: a randomized controlled trial to investigate changes 
in intrinsic brain connectivity following Cognitive Behavioral Therapy. NeuroImage: Clinical 2014;5:365-376.

45. Smith SM, Fox PT, Miller KL, Glahn DC, Fox PM, Mackay CE, Filippini N, Watkins KE, Toro R, Laird AR, and Beckmann CF. Correspondence of the brain's functional architecture during activation and rest. Proc.Natl.Acad.Sci.U.S.A 2009;106(31):13040-13045.

46. Sullivan MJ, Bishop SR, and Pivik J. The pain catastrophizing scale: development and validation. Psychological Assessment 1995;7(4):524-532.

47. Thorn B, Cognitive therapy for chronic pain: a step-by-step guide. 2004, New York: Guilford Press.

48. Turk DC, Meichenbaum D, and Genest M, Pain and behavioral medicine: a cognitive behavioral perspective. 1983, New York: Guilford Press.

49. Upadhyay J, Lemme J, Anderson J, Bleakman D, Large T, Evelhoch JL, Hargreaves R, Borsook D, and Becerra L. Test-retest reliability of evoked heat stimulation BOLD fMRI. J Neurosci Methods 2015;253:38-46.

50. Wager TD and Atlas LY. The neuroscience of placebo effects: connecting context, learning and health. Nat Rev Neurosci 2015;16(7):403-18.

51. Weissman-Fogel I, Moayedi M, Tenenbaum HC, Goldberg MB, Freeman BV, and Davis KD. Abnormal cortical activity in patients with temporomandibular disorder evoked by cognitive and emotional tasks. Pain 2011;152(2):384-396.

52. Wiech K, Kalisch R, Weiskopf N, Pleger B, Stephan KE, and Dolan RJ. Anterolateral prefrontal cortex mediates the analgesic effect of expected and perceived control over pain. J Neurosci 2006;26(44):11501-9.

53. Wiech K, Ploner M, and Tracey I. Neurocognitive aspects of pain perception. Trends Cogn Sci. 2008;12(8):306-313.

54. Williams AC, Eccleston C, and Morley S. Psychological therapies for the management of chronic pain (excluding headache) in adults. Cochrane Database Syst Rev 2012;11:CD007407.

55. Woo CW, Roy M, Buhle JT, and Wager TD. Distinct brain systems mediate the effects of nociceptive input and self-regulation on pain. PLoS Biol 2015;13(1):e1002036.

56. Worsley KJ, Liao CH, Aston J, Petre V, Duncan GH, Morales F, and Evans AC. A general statistical analysis for fMRI data. NeuroImage 2002;15(1):1-15.

57. Yeo BT, Krienen FM, Sepulcre J, Sabuncu MR, Lashkari D, Hollinshead M, Roffman JL, Smoller JW, Zollei L, Polimeni JR, Fischl B, Liu H, and Buckner RL. The organization of the human cerebral cortex estimated by intrinsic functional connectivity. J Neurophysiol 2011;106(3):1125-65.

58. Yoshimura S, Okamoto Y, Onoda K, Matsunaga M, Okada G, Kunisato Y, Yoshino A, Ueda K, Suzuki S, and Yamawaki S. Cognitive behavioral therapy for depression changes medial prefrontal and ventral anterior cingulate cortex activity associated with self-referential processing. Soc Cogn Affect Neurosci 2014;9(4):487-93.

59. Zeidan F, Emerson NM, Farris SR, Ray JN, Jung Y, McHaffie JG, and Coghill RC. Mindfulness Meditation-Based Pain Relief Employs Different Neural Mechanisms Than Placebo and Sham Mindfulness Meditation-Induced Analgesia. J Neurosci 2015;35(46):15307-25. 


\section{Figure Legends}

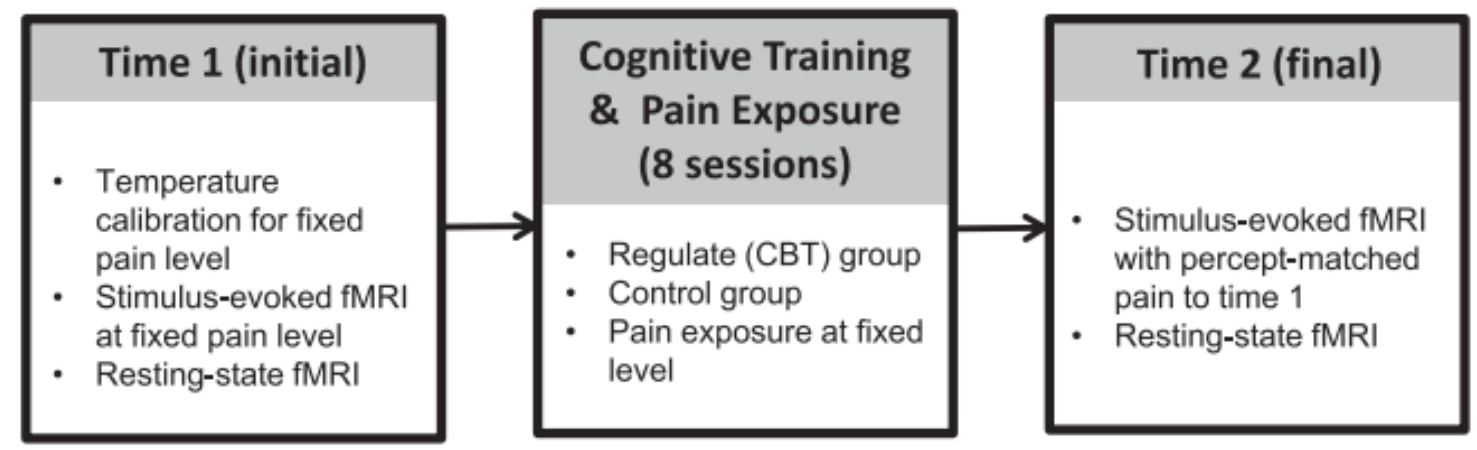

Figure 1

An overview of the study paradigm. Subjects underwent pre-intervention neuroimaging, including fMRI with painful stimulation and at rest, and were then either assigned to a Regulate (CBT) or (active) Control group. Over 8 sessions on different days, subjects in each group received cognitive training and were exposed to pain. After training, subjects returned for a second neuroimaging session, including fMRI with painful stimulation percept-matched to the initial session and at rest. CBT $=$ cognitive behavioral training. 

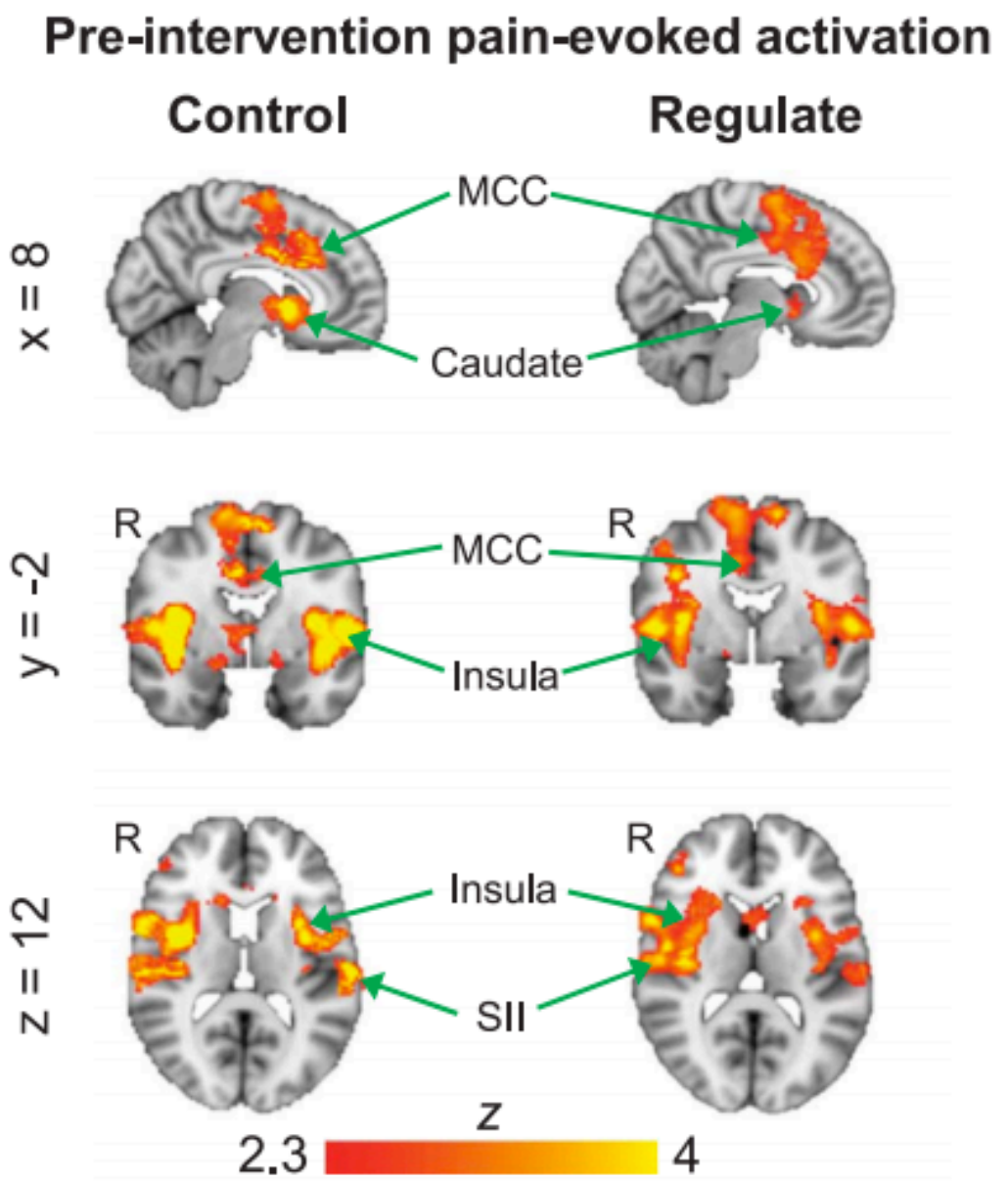

\section{Pre-intervention pain-evoked deactivation Control \\ Regulate}
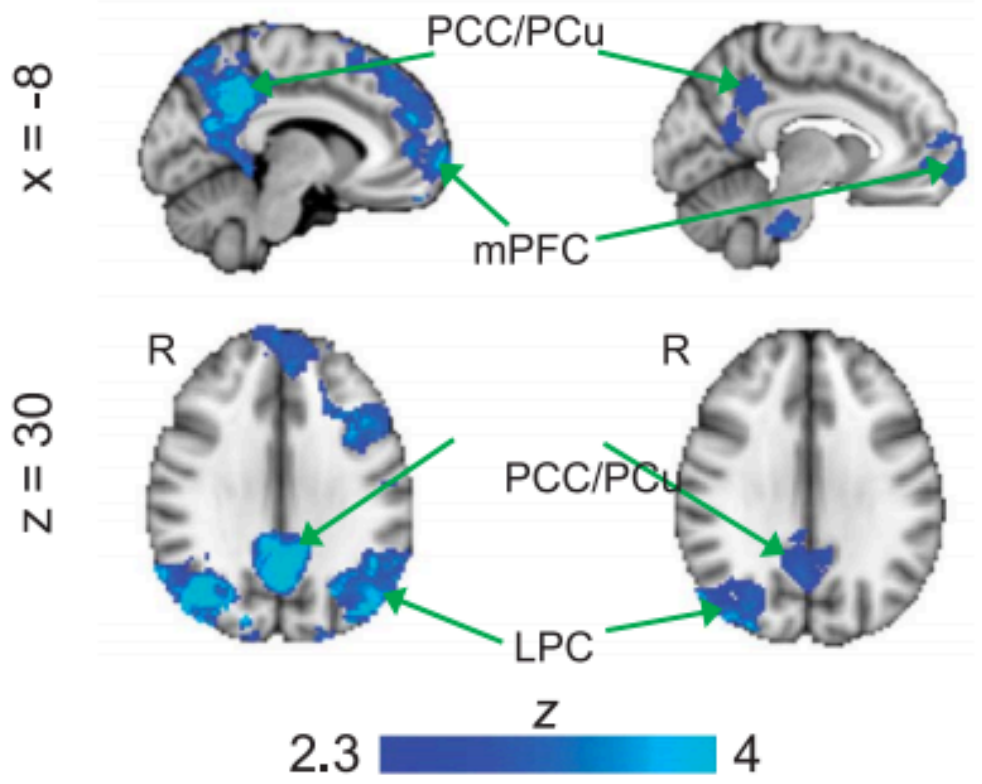


\section{Figure 2}

Pre-intervention brain activation and deactivation during painful stimulation in the Control and Regulate group. Both groups show activation in salience- (e.g. insula, MCC) and sensory-related (e.g. SII) regions and deactivation in regions of default mode network (e.g. $\mathrm{mPFC}, \mathrm{PCC}$ ) (FWE-corrected $\mathrm{Z}>2.3$, cluster-based $\mathrm{p}<0.05$ ). No significant group differences in brain activation/deactivation were found. LPC = lateral parietal cortex; $\mathrm{MCC}=$ mid-cingulate cortex $\mathrm{mPFC}=$ medial prefrontal cortex $; \mathrm{PCC}=$ posterior cingulate cortex; $\mathrm{PCu}=$ precuneus. 
A Control group: decreased DMN deactivation over time

Time $2>$ Time 1

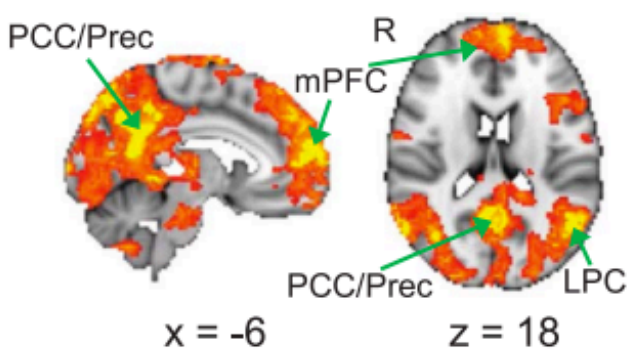

Regulate group: decreased dIPFC/vIPFC activation over time Time $2>$ Time 1

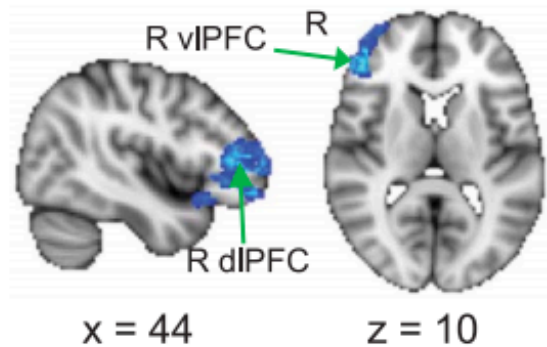

C Group differences in pain-evoked brain activation/deactivation changes over time
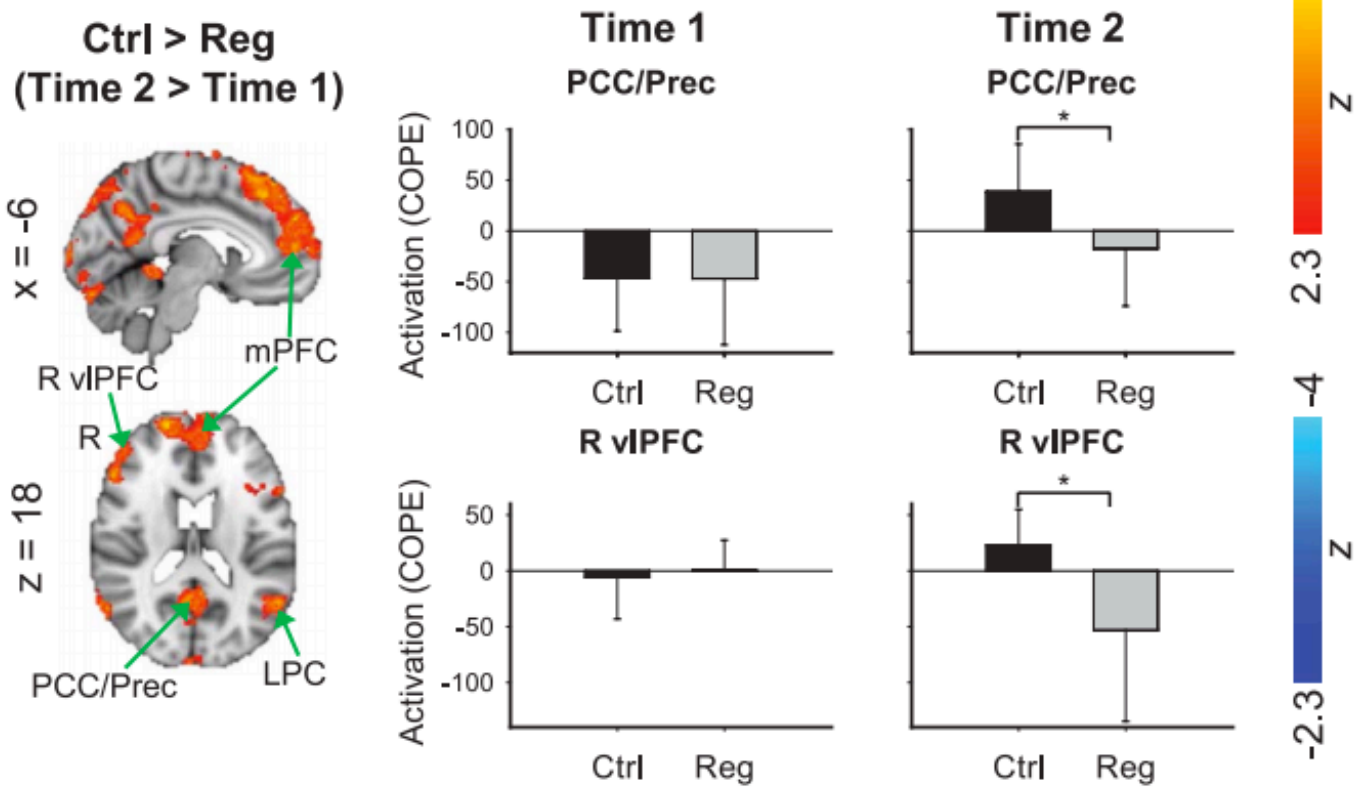

\section{Figure 3}

Changes in brain activation during painful stimulation after cognitive training compared to before training. A) The Control group largely showed decreases in deactivation of default mode network areas over time; B) The Regulate showed decreased activation of dorso-/ventro-lateral prefrontal cortical regions over time; C) A group comparison revealed greater activation changes over time in Control compared to Regulate group in default mode and lateral prefrontal regions (all maps displayed at FWE-corrected $\mathrm{Z}>2.3$; 
cluster-based $\mathrm{p}<0.05)$. $\mathrm{CBT}=$ cognitive behavioral training; $\mathrm{Ctrl}=$ control; $\mathrm{dlPFC}=$ dorsolateral prefrontal cortex; $\mathrm{DMN}=$ default mode network; $\mathrm{LPC}=$ lateral parietal cortex MCC $=$ mid-cingulate cortex $; \mathrm{mPFC}=$ medial prefrontal $\operatorname{cortex} ; \mathrm{PCC}=$ posterior cingulate cortex $;$ Prec $=$ precuneus $;$ Reg $=$ regulate $; \mathrm{vlPFC}=$ ventrolateral prefrontal cortex. 


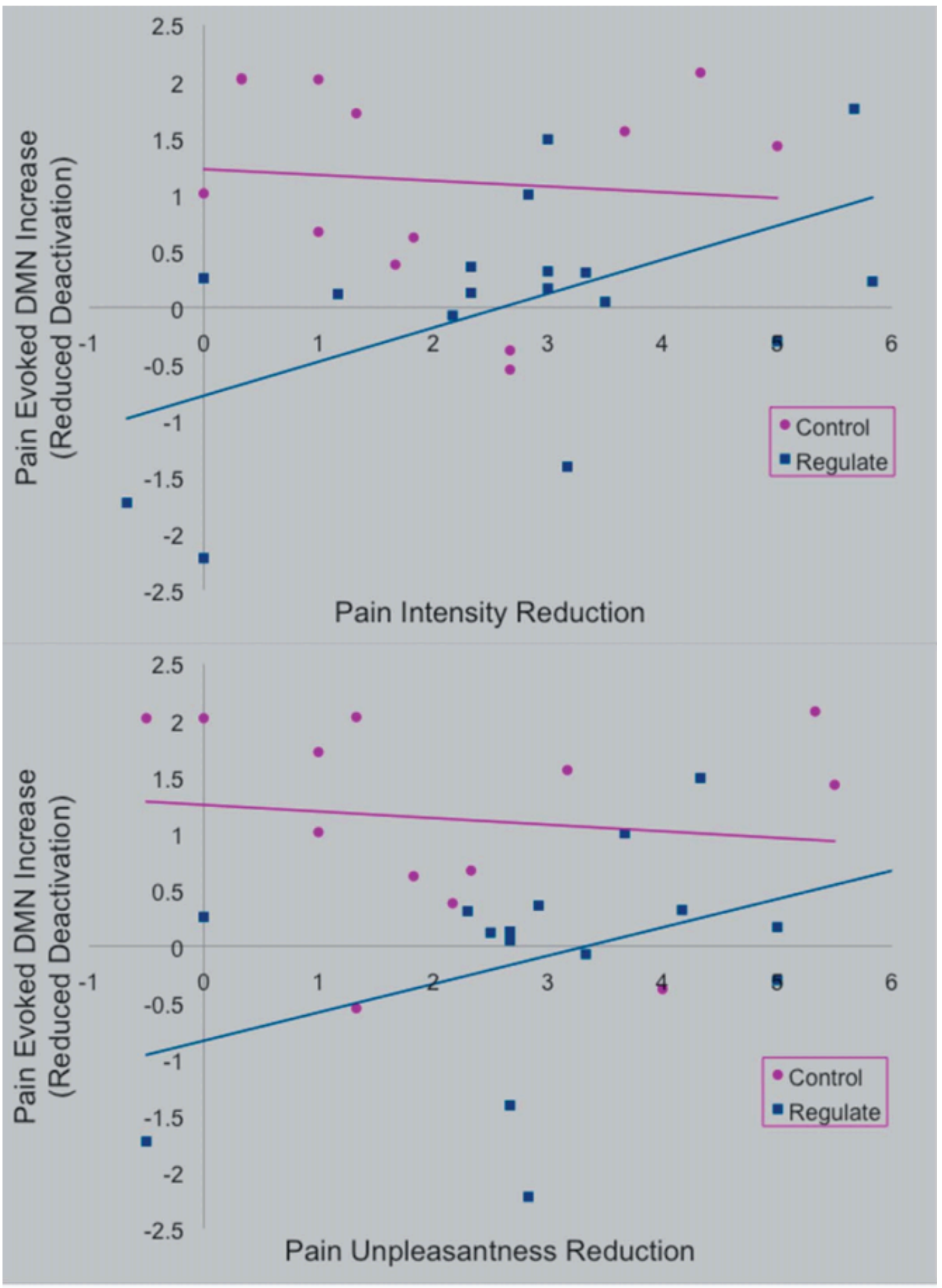

Figure 4 
Relationships of reduced pain intensity and unpleasantness over time (measured in behavioral sessions) and pain-evoked default mode network (DMN) activity (measured during fMRI) in Control and Regulate groups. In the Regulate group there was a positive correlation of reduced pain-evoked DMN deactivation at the final compared to first scan with reduced pain intensity $(r=0.48, p=0.051)$ and unpleasantness $(r=0.5, p=0.04)$ at the last compared to first behavioral session. In the Control group, there were no such correlations with pain intensity $(\mathrm{r}=0.12, \mathrm{p}=0.71)$ or unpleasantness $(\mathrm{r}=0.02, \mathrm{p}=0.96)$.

\section{Pre-intervention resting $\mathrm{R}$ vIPFC FC}

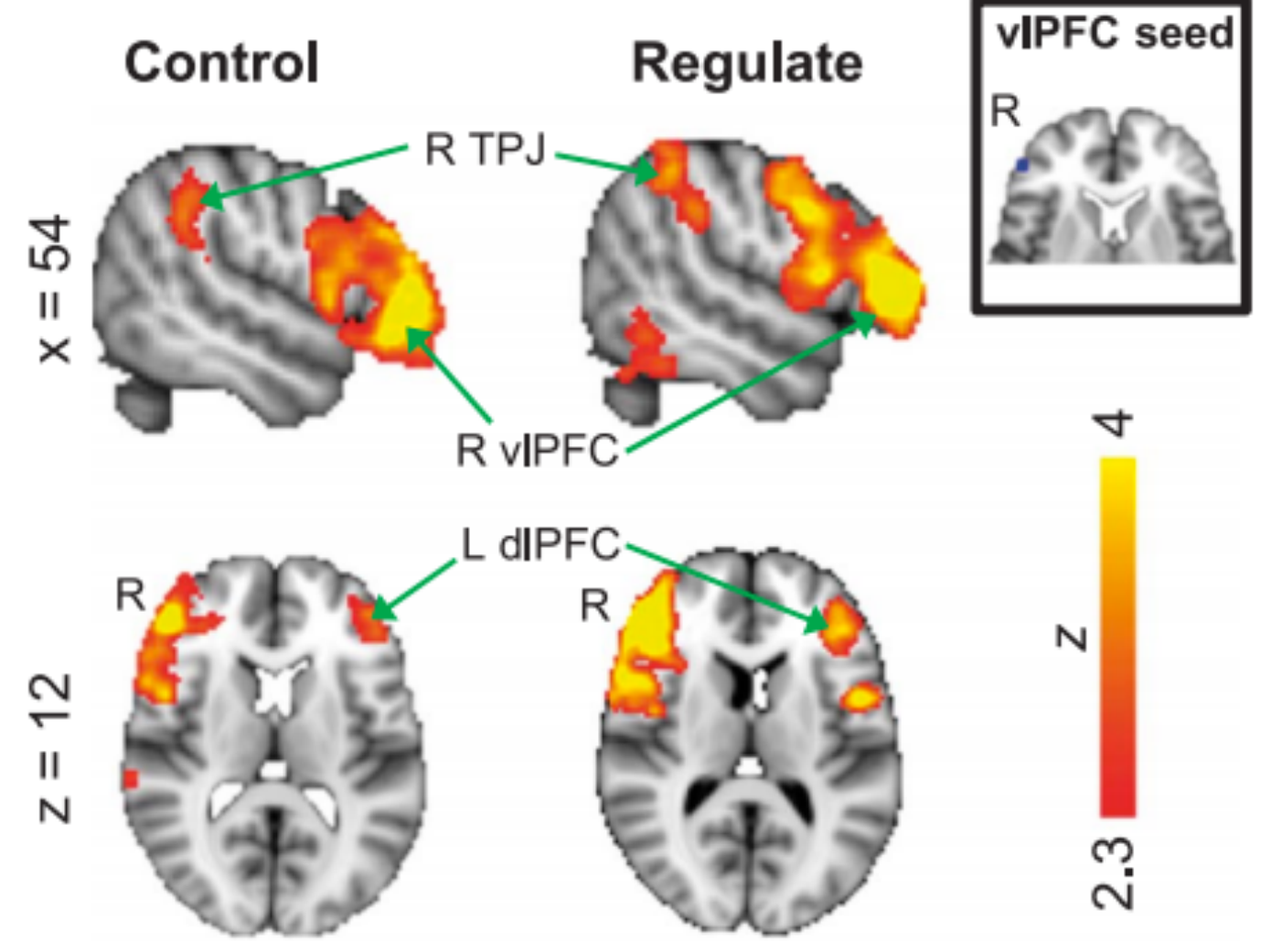

\section{Figure 5}

Pre-intervention resting-state functional connectivity of right ventrolateral prefrontal cortex (R vlPFC) in the Control and Regulate group. Both groups show functional 
connectivity with regions in the executive control network (e.g. dIPFC, TPJ) (FWEcorrected $\mathrm{Z}>2.3$, cluster-based $\mathrm{p}<0.05$ ). No significant group differences in resting $\mathrm{R}$ vlPFC functional connectivity were found $\mathrm{Ctrl}=$ control; $\mathrm{dlPFC}=$ dorsolateral prefrontal cortex; FC = functional connectivity; Reg = regulate; TPJ = teomporoparietal junction; $\mathrm{vlPFC}=$ ventrolateral prefrontal cortex.

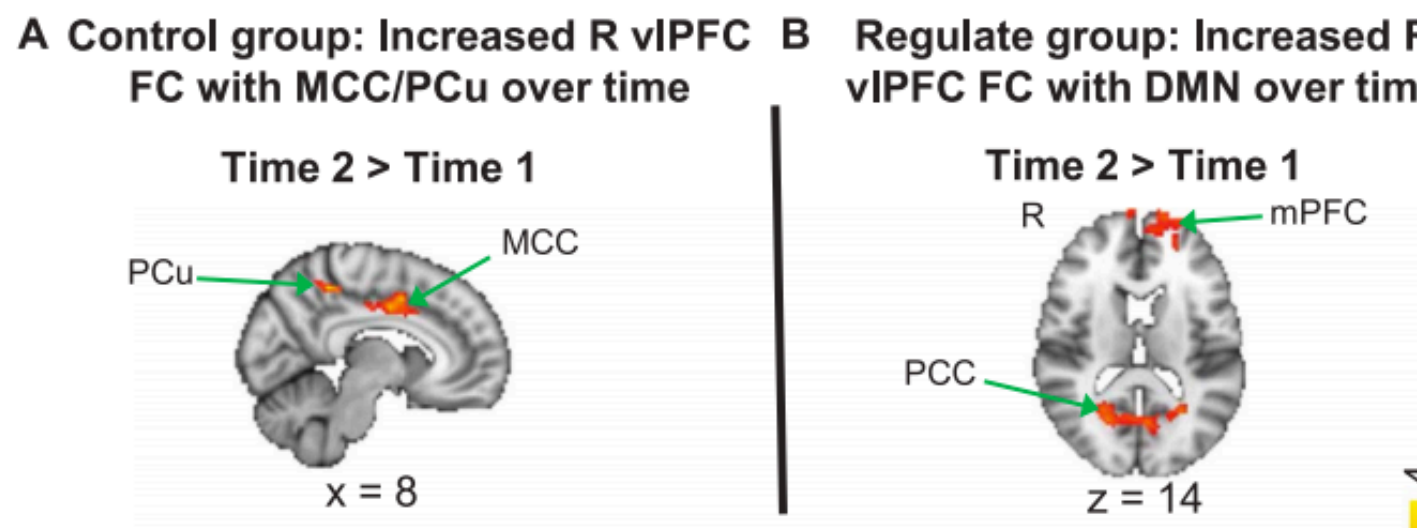

C Greater increase in R vIPFC resting FC over time in Regulate Ctrl $>$ Reg compared to Control group (Time $2>$ Time 1)
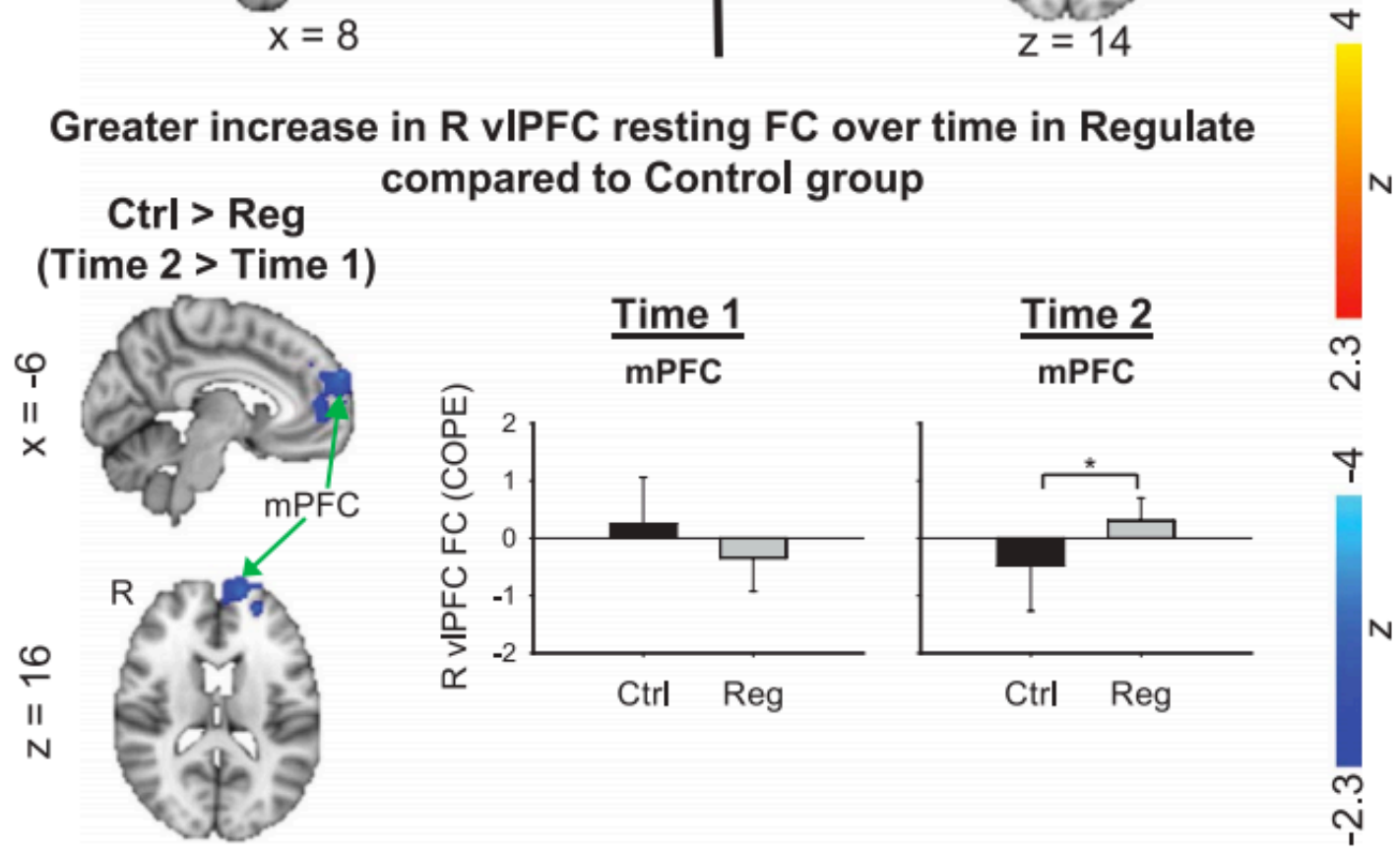

Figure 6

Changes in resting R vlPFC functional connectivity after cognitive training compared to before training. A) The Control group showed increased functional connectivity with the MCC and PCu over time; B) The CBT (Reg) group showed increased functional 
connectivity with default mode network areas (PCC, mPFC) over time; C) A group comparison revealed a greater increase in functional connectivity with the $\mathrm{mPFC}$ in the CBT compared to Control group over time (all maps displayed at FWE-corrected $\mathrm{Z}>2.3$; cluster-based $\mathrm{p}<0.05) . \mathrm{Ctrl}=$ control; $\mathrm{dlPFC}=$ dorsolateral prefrontal cortex; $\mathrm{DMN}=$ default mode network; $\mathrm{FC}$ = functional connectivity; $\mathrm{MCC}=$ mid-cingulate cortex; $\mathrm{mPFC}=$ medial prefrontal cortex; $\mathrm{PCC}=$ posterior cingulate cortex $\mathrm{PCu}=$ precuneus; Reg $=$ regulate $; \mathrm{vlPFC}=$ ventrolateral prefrontal cortex.

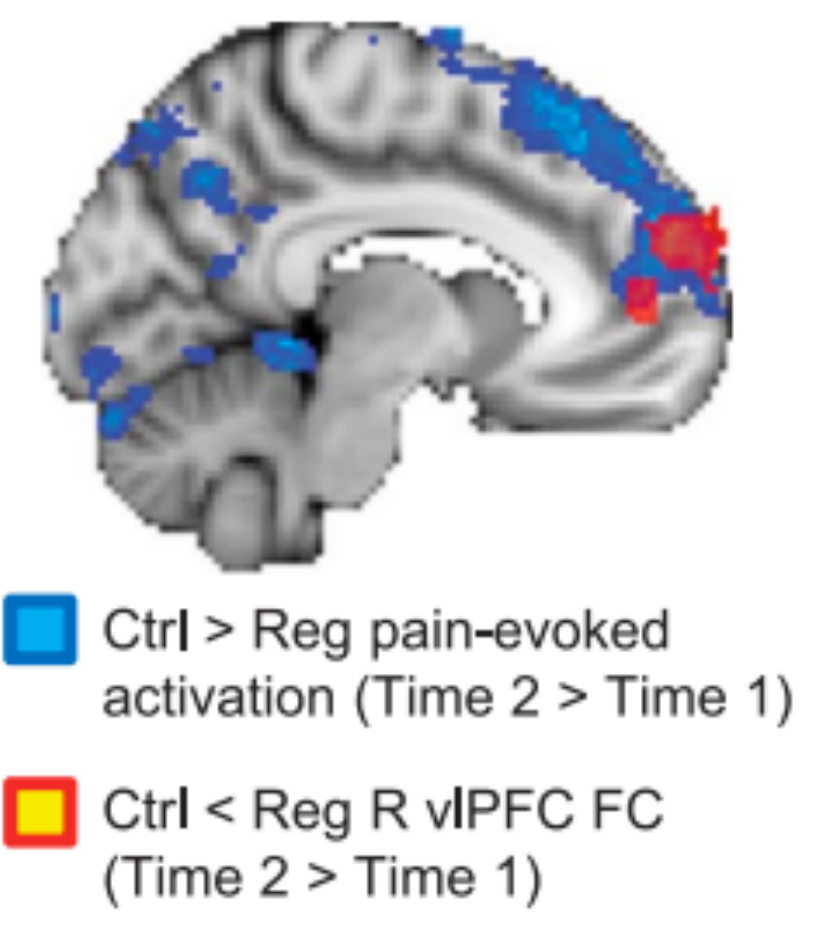

Figure 7

An overlay of regions showing Control $>$ Regulate activation during painful stimulation over time (blue) and regions showing Control > Regulate resting-state functional connectivity of the R vlPFC (red/yellow) over time. A common cluster within the medial prefrontal cortex was significant in both analyses (both maps displayed at FWE-corrected 
$\mathrm{Z}>2.3$, cluster-based $\mathrm{p}<0.05) . \mathrm{CBT}=$ cognitive behavioral training; $\mathrm{Ctrl}=$ control; $\mathrm{FC}=$ functional connectivity; Reg $=$ regulate; $\mathrm{vlPFC}=$ ventrolateral prefrontal cortex. 